\title{
Effects of antioxidant supplements consumed at night on endothelial function in healthy volunteers
}

\author{
R. Patel, M. Gibbs and S. Hampton \\ Faculty of Health and Medical Sciences, University of Surrey, Guildford, UK
}

The endothelium is an active tissue; it performs many anti-atherogenic functions and its functional efficacy is an important factor in CVD risk. Epidemiological data suggest that there is a peak incidence of cardiovascular events during the early morning ${ }^{(1)}$. The existence of a $24 \mathrm{~h}$ circadian rhythm in endothelial function, which is attenuated in the early morning, is one potential factor for this peak in incidence ${ }^{(2)}$. Recently, dietary intake and nutritional supplementation have been shown to have effects on endothelial function. The aim of the present project was to investigate the effect of 6 weeks of antioxidant supplements taken at night, in the form of multivitamin and mineral tablets, on morning endothelial function. It is hypothesised that night-time antioxidant supplementation will improve early-morning endothelial function.

A range of nutrition supplements widely available in health food shops were assessed for antioxidant capacity before the start of the study. Seven healthy volunteers were recruited (two males and five females; mean age 24.1 (SE 7); BMI 21.6 (SE 1.8 ) kg/m²; non-smokers; free from prescribed medication and nutritional supplements). They were instructed to consume an ABC plus multivitamin and multimineral formula tablet (Holland and Barrett, Nuneaton, War., UK), antioxidant level $250 \mu \mathrm{mol}$ Trolox per tablet, at 21.00 hours every evening. One subject was excluded on the basis of measurement precision. Endothelial function was assessed by measuring flow-mediated dilatation (FMD) of the brachial artery using an ultrasound technique. Resting arterial diameter (AD) was measured at baseline and post treatment to ensure measurement occurred in the same position on the brachial artery. FMD, blood glucose, NEFA and TAG were assessed at pretreatment and after 6 weeks of supplementation.

The Table shows baseline AD and percentage FMD pre- and post supplementation. Mean percentage FMD increased by $44 \%$ from pretreatment $(5.39$ (SE 0.61)) to week $6(7.78$ (SE 2.15)) but the increase was not significant $(P=0.25)$. Resting AD pre- and post treatment were similar (3.42 (SE 0.11) v. 3.43 (SE 2.15); NS). No significant changes in plasma glucose, TAG or NEFA levels were observed over 6 weeks.

\begin{tabular}{llllc}
\hline \multirow{2}{*}{ Subjects } & \multicolumn{2}{c}{ Pretreatment } & & \multicolumn{2}{c}{6 weeks post treatment } \\
\cline { 2 - 3 } \cline { 5 - 5 } & AD $(\mathrm{mm})$ & A FMD & & ADm) FMD \\
\hline 1 & 3.54 & 6.60 & 3.21 & 16.9 \\
2 & 3.64 & 4.10 & 3.64 & 4.66 \\
3 & 3.78 & 6.86 & 3.68 & 4.37 \\
4 & 3.10 & 3.57 & 3.05 & 2.89 \\
5 & 3.12 & 6.75 & 3.20 & 10.8 \\
7 & 3.35 & 4.47 & 3.81 & 7.02 \\
Mean & $3.42(\mathrm{SE} 0.11)$ & $5.39(\mathrm{SE} 0.61)$ & $3.43(\mathrm{SE} 0.13)$ & 7.78 (SE 2.15) \\
\hline
\end{tabular}

In the present small pilot study antioxidant supplementation in the form of multivitamin and multimineral tablets showed a slight improvement in morning endothelial function. Four subjects showed an improvement while two subjects showed a reduction in FMD. This finding suggests a possible future role for antioxidant supplementation in reducing early-morning cardiovascular events by improved endothelial function at this time of increased risk. However, further investigation is needed with a larger sample size, varying sample dose and perhaps alternative methods of assessing endothelial function.

1. Muller JE, Stone PH, Turi ZG et al. (1985) N Engl J Med 313, 1315-1322.

2. Walters J, Skene D, Hampton SM \& Ferns GA (2003) Med Sci Monit 9, RA1-RA8. 